\title{
Case Report \\ OPen Access \\ Intraperitoneal escape of kidney stones during laparoscopic pyelolithotomy: case report
}

CrossMark

\begin{abstract}
Endourology is the main treatment modality used for urinary stones, Laparoscopy has limited role in special situations and little is written about its complications. We present a case of lost renal stones during transperitoneal laparoscopic pyelolithotomy (LP) managed conservatively followed by successful removal of stones my small low midline incision.
\end{abstract}

Keywords: laparoscopic pyelolithotomy, lost stones, urolithiasis
Volume 8 Issue 6 - 2020

\section{Mohammed Mahdi Babakri}

Faculty of Medicine, Aden University, Yemen

Correspondence: Mohammed Mahdi Babakri, Faculty of Medicine, Aden University, Raidiand Specilaized Clinic, Aden, Yemen, Email drbabakri@gmail.com
Abbreviations: LP, laparoscopic pyelolithotomy; SWL, shock wave lithotripsy; PCNL, percutanous nephrolithotomy

\section{Introduction}

Management of urinary stones witnessed dramatic changes during the last few decades supported by rapidly developing technologies. Laparoscopy has been adopted for management of many urological disorders, the indications for laparoscopic management of urinary stones are few ${ }^{1}$ for that reason few articles has been published describing the laparoscopic management of urinary stones. ${ }^{2}$ Many authors have reported cases of lost gall stones ${ }^{3}$ and described the fate and complications of it. By searching the literature only one article has reported an intraperitoneal loss of kidney stone presented as adnexal mass three years after laparoscopic nephrectomy. ${ }^{4}$ We reported the first case of intraperitoneally lost kidney stones after laparoscopic peylolithotmoy.

\section{Case presentation}

45 years old male patient came to urology outpatient complaining of recurrent right flank pain for several months, no fever or hematuria, no significant medical or surgical history. Physical examination was unremarkable, serum creatinin $1.1 \mathrm{mg} / \mathrm{dl}$, urine analysis showed microscopic hematuria, urine culture was negative, ultrasound reveals multiple right renal stones with dilated extrarenal pelvis, plain $\mathrm{x}$ ray \& intravenous pyelography performed Figure1 (A \& B). All treatment options explained to the patient and after discussion he elected to undergo laparoscopic peylolithotomy. Transperitoneal laparoscopic pyelolithotomy was performed in lateral position with Rt. Flank tilted 45 degrees using 4 ports. multiple renal pelvis and calyceal stones extracted and putted in a bag made of No 6 surgical glove (Figure 2) after closure of the pyelotomy by intracorporeal sutures the bag containing the stones extracted through the umbilical port after enlarging it (Figure 2), during extraction the bag was broken and some stones escaped to the abdomen. Another bag replaced and most of the stones extracted searching for the remaining stones in the abdomen was frustrating and we decided to terminate the procedure and closing the port sites. The post-operative course is unremarkable and patient discharged in fifth post-operative day. Follow up plain x-ray after one month revealed tow round stones with 30 and $10 \mathrm{~mm}$ maximum diameter lying deep in the pelvis (Figure 3), after discussion with the patient about the possible complications of those stones he agree to underwent open extraction by low midline incision and stones extracted.

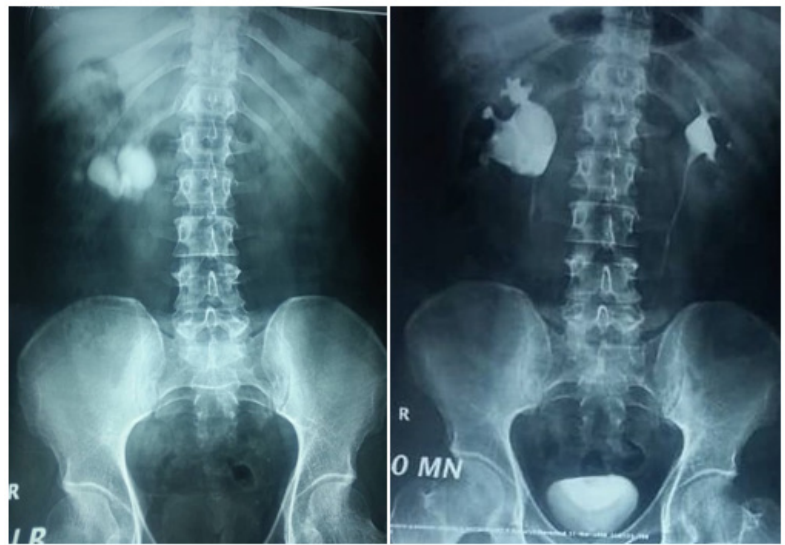

Figure I Pre operative Pain abdominal $x$ ray $(A)$ and intravenous pyelography (B).

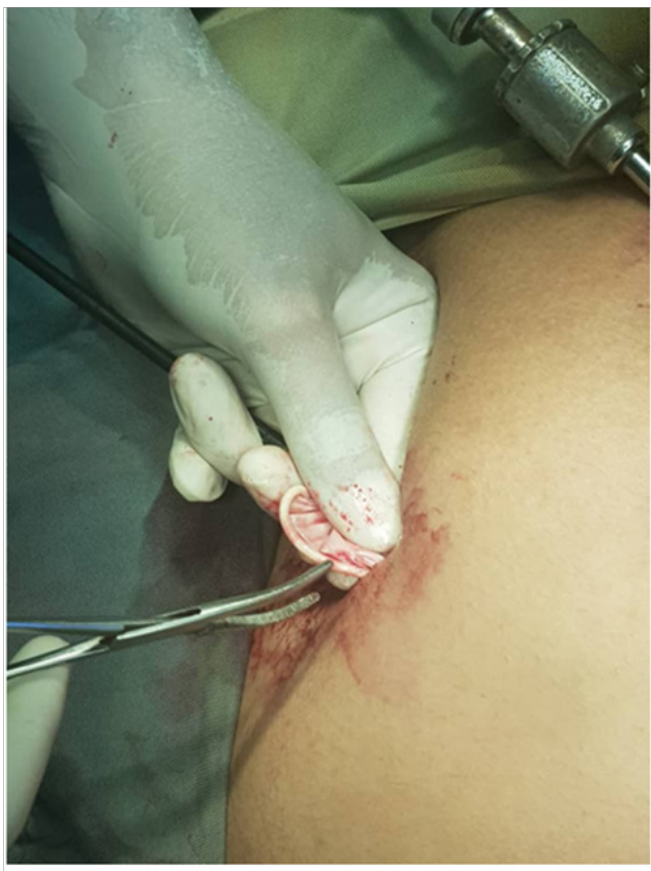

Figure 2 Custom made retrieval bag made of No 6 surgical glove. 


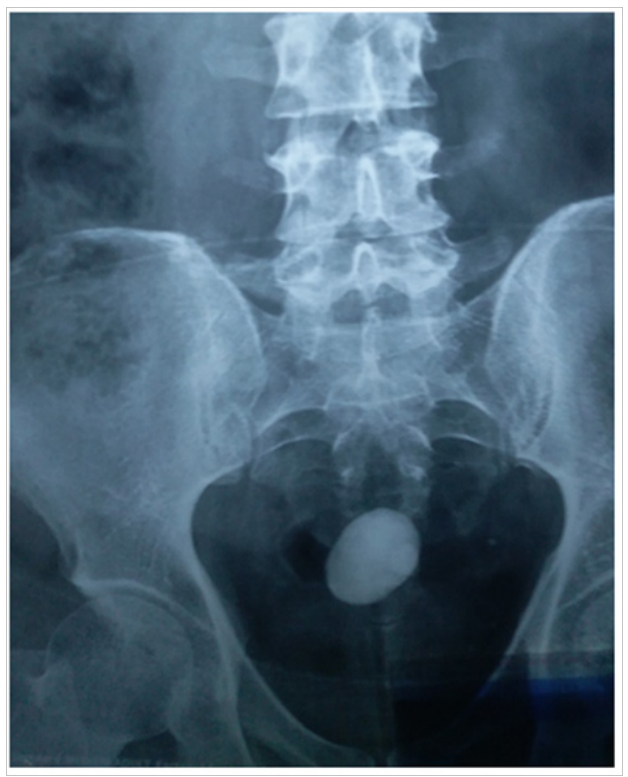

Figure 3 Post operative $x$ ray showing lost stones in the pelvis.

\section{Discussion}

The evolution of renal stones surgery had rendered open surgery almost obsolete, there are special situations where endourology and shock wave lithotripsy (SWL) is not ideal for managing urinary stones (e.g. stone in ectopic kidney, PUJO), and laparoscopy has replaced traditional open surgery in these special situations. Compared to Percutanous nephrolithotomy (PCNL) laparoscopic pyelolithotomy has more stone free rate and less bleeding and longer operative time. ${ }^{5}$ There are many report about lost gall bladder stones after laparoscopic cholecystectomy describing a lot of consequences such as abscess foreign body granuloma, fistula, bowel obstruction...etc ${ }^{3}$., however by searching the major medical data bases we found on case report of lost kidney stone after laparoscopic nephrectomy presented three years later as adnexal mass. ${ }^{4}$ Carful stone manipulation and gentle extraction is crucial to prevent stones from escape in the abdomen. To the best of our knowledge we report the first case of intraperitonealy lost kidney stones following laparoscopic pyelolithotomy, as there is a lake of guidelines regarding the management of this rare complication and the significantly large size of the lost stone we decided to remove it.

\section{Acknowledgments}

None.

\section{Consent}

Informed consent was obtained from the patient for use of his clinical information and clinical photos.

\section{Declarations interest}

The authors report no conflict of interests.

\section{Funding}

This research did not receive any specific grant from funding agencies in the public, commercial, or not-for-profit sectors.

\section{References}

1. Türk C, Petrrík A, Sarica K, et al. EAU guidelines on interventional treatment for urolithiasis. Eur Urol. 2016;69(3):475-482.

2. Stein RJ, Turna B, Nguyen MM, et al. Laparoscopic pyeloplasty with concomitant pyelolithotomy: Technique and outcomes. $J$ Endourol. 2008;22:1251-1255.

3. T Sathesh-Kumar, A P Saklani, R Vinayagam, et al. Spilled gall stones during laparoscopic cholecystectomy: a review of the literature. Postgrad Med J. 2004;80:77-79.

4. Barone JE, Kardon S. An Unusual Complication of a Laparoscopic Nephrectomy: The "Lost" Kidney Stone', Urology. Elsevier Inc. 2009;73(5):1164.e1-1164.e3.

5. Zhao C, Yang H, Tang K, et al. Comparison of laparoscopic stone surgery and percutaneous nephrolithotomy in the management of large upper urinary stones: A meta-analysis. Urolithiasis. 2016;44:479-490. 